\title{
Estimation Direction Arrival of Smart Antenna
}

\author{
Adiba EL Fadl ${ }^{1}$, Mohammed Amine Ihedrane ${ }^{2}$, Seddik Bri ${ }^{2}$ \\ ${ }^{1}$ Physics Department, Normal School Superior, Mohamed V University, Rabat - Morocco \\ adifadl@gmail.com \\ ${ }^{2}$ MIN Group, High School of Technology, Moulay Ismail University - Meknes-Morocco \\ amine.ihedrane@gmail.com, briseddik@gmail.com
}

\begin{abstract}
Smart antenna has been universally used in many applications due to their merits of great channel capacity, reducing the co-channel interferences for deployment and information implementation for communication technologies in smart cities. This kind of antenna is used in radar, sonar and wireless communication systems. In this article we expounds in detail how to obtain Direction of Arrival (DOA) information using Multiple Signal Classification (MUSIC) algorithm with Uniform Circular Arrays (UCA) based on theoretical analysis of receiving spatial signal by reconstruction of the data matrix. Simulations results are compared with the experimented measures published. This comparison demonstrates that the proposed MUSIC algorithm is more accurate and stable.
\end{abstract}

Key words : About four key words or phrases in alphabetical order, separated by commas.

\section{INTRODUCTION}

Smart antenna has widely used in varieties applications like mobile communications, sonar [1].

DOA estimation uses antenna arrays. It is known that antenna radiation main lobe beam width is inversely proportional to the number of elements in antenna. So, if we consider a single antenna then array pattern will be wider and the resolution cannot be good. Instead of using single antenna, an antenna array system is used in DOA estimation which will improve the resolution of the received signals (Resolution in DOA estimation is the ability to distinguish two signals arriving at different angles). An array system has a multiple elements distributed in space [2].so spectrum is a natural resource that enables us to communicate and exchange our data over wireless channels [3-5].

One major limitation of this method is poor resolution that is its ability to separate closely spaced signals. Unlike conventional methods, subspace methods exploit the information of the received data resulting in high resolution. Two main subspace based algorithms are Multiple Signal Classification and Estimation of Signal Parameters with
Rotational Invariance Techniques. The basic concept of the DOA estimation is to focus/maximize the reception of the estimated/main direction and to reject all the received interferences from other directions. Numerous techniques have been established to estimate the signal DOA, such as the conventional techniques (Bartlett and Capon), subspace based techniques (e.g. ESPRIT (Estimation of Signal Parameters via Rotational Invariance Techniques) and the MUSIC (Multiple Signal Classification), maximum likelihood techniques, integrated techniques and local polynomial approximation beam-former.

The MUSIC algorithm is applied and has high resolution estimation, due to the eigen-structure of the data covariance matrix of the receive array. In conventional subspace approaches in MIMO radar system, we usually assume that the number of array elements remains fixed and the number of snapshots is large which could be infinity. In such situation, the statistic covariance matrix of the observed signals could be substituted by their sample covariance matrix (SCM) to obtain accurate target parameters. However, in large MIMO radar system [6-11] in which the number of the transmitter and receiver array elements is so large that the number of observations is unable to satisfy the demand given above.

This article expounds in detail how to obtain DOA information using a modified MUSIC algorithm through re-construction data matrix with conjugate data. The comparison between proposed method and the experimented one. The proposed method can solve the problem of coherent signals and estimate clearly the direction of arrival.

\section{2-D MUSIC ALGORITHM}

Multiple Signal Classification (MUSIC) is an approach was first posed by Schmidt [9]. It is well known for the implementation simplicity as well as the capability of estimating DOA in much higher resolution than any other conventional algorithms [12-16]. We assume that there are $\mathrm{N}$ uniform circular array, $\mathrm{M}$ narrow band far field signals from different incident direction. The radius of the circular array is denoted $r$ and wavelength of narrow band $\lambda$. The incident angle of the signals is shown in Figure 1. 


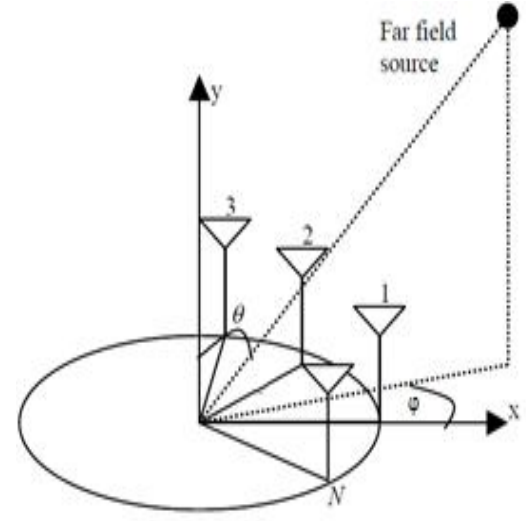

Figure 1: UCA geometry

The received array signal can be described as :

$$
X(t)=A S(t)+N(t)
$$

Where $A=\left[a\left(\theta_{1}, \varphi_{1}\right), \ldots, a\left(\theta_{M}, \varphi_{M}\right)\right]$ is a matrix of the $M$ steering vectors, which represents the possible value set of DOA. $\mathrm{a}(\theta, \varphi)=\left[\mathrm{e}^{\mathrm{j} 2 \pi_{\lambda}^{\mathrm{r}} \sin \varphi_{\mathrm{m}} \cos \theta_{\mathrm{m}}}, \ldots, \mathrm{e}^{\mathrm{j} 2 \pi_{\lambda}^{\mathrm{r}} \sin \varphi_{\mathrm{m}} \cos \left(\theta_{\mathrm{m}}-\frac{2 \pi(\mathrm{N}-1)}{\mathrm{N}}\right)}\right]$ and $\mathrm{S}=[\mathrm{S} 1(\mathrm{t}), \ldots, \mathrm{SM}(\mathrm{t})]^{\mathrm{T}}$ is a signal source vector of size ( $\mathrm{M} \times 1)$. Where the statistical expectation is denoted by $\mathrm{E}$ [ ] and []$^{\mathrm{T}}$ denote transpose of signal vector. The correlation matrix of received vector can be written as:

$$
\mathrm{Rx}=\mathrm{E}\left[\mathrm{XX}^{\mathrm{H}}\right]=\mathrm{AVA}^{\mathrm{H}}+\sigma^{2} \mathrm{I}=\mathrm{R}_{\mathrm{s}}+\sigma^{2} \mathrm{I}
$$

Where $\sigma^{2}$ is the variance of white Gaussian noise vector (W), $\mathrm{V}$ is covariance matrix of signal vector $(\mathrm{S})$ which is a full rank matrix of order $\mathrm{M} \times \mathrm{M}$ given by :

$$
\begin{aligned}
\mathrm{V} & =\mathrm{E}\left[\mathrm{SS}^{\mathrm{H}}\right] \\
& =\left[\begin{array}{cccc}
\mathrm{E}\left[\left.\mathrm{S}_{1}\right|^{2}\right] & \ldots & \ldots & 0 \\
0 & \left.\left.\mathrm{E}^{[} \mathrm{S}_{2}\right|^{2}\right] & \ldots & 0 \\
\vdots & \ddots & \ldots & \vdots \\
0 & 0 & \ldots & \mathrm{E}\left[\left.\mathrm{S}_{\mathrm{M}}\right|^{2}\right]
\end{array}\right]
\end{aligned}
$$

$R_{S}$ is a signal covariance matrix of order $(N \times N)$ with rank $M$ given by:

$$
\mathrm{R}_{\mathrm{S}}=\left[\begin{array}{cccccc}
\mathrm{E}\left[\left|\mathrm{S}_{1}\right|^{2}\right] & \ldots & \ldots & 0 & \ldots & 0 \\
0 & \mathrm{E}\left[\left|\mathrm{S}_{2}\right|^{2}\right] & \ldots & 0 & \ldots & 0 \\
\vdots & \ddots & \ldots & \vdots & . & 0 \\
0 & 0 & \ldots & \left.\left.\mathrm{E}^{\mid} \mathrm{S}_{\mathrm{M}}\right|^{2}\right] & \ldots & 0 \\
0 & 0 & \ldots & 0 & \ldots & 0
\end{array}\right]
$$

So $R_{S}$, has N-M eigenvectors corresponding to zero eigen values. We know that steering vector a $(\theta, \varphi)$ which is in the signal subspace is orthogonal to noise subspace let $\mathrm{Q}_{\mathrm{n}}$ be such an eigenvector.

$$
\mathrm{R}_{\mathrm{S}} \mathrm{Q}_{\mathrm{n}}=\mathrm{AVA}^{\mathrm{H}} \mathrm{Q}_{\mathrm{n}}=0
$$

Since $\mathrm{V}$ is a positive definite matrix:

$$
\mathrm{a}^{\mathrm{H}}\left(\theta_{\mathrm{i}}, \varphi_{\mathrm{i}}\right) \mathrm{Q}_{\mathrm{n}}=0
$$

This implies that signal steering vectors are orthogonal to eigen vector corresponding to noise subspace. So the MUSIC algorithm searches through all angles and plots the spatial spectrum:

$$
\mathrm{P}_{\text {MUSIC }}(\theta, \varphi)=\frac{1}{\left({ }^{\mathrm{H}}(\theta, \varphi) Q_{n} Q_{n}{ }^{H} \mathrm{a}(\theta, \varphi)\right)}
$$

\section{PROPOSED METHOD}

In order to have a better estimation of direction of arrival, we have proposed a modified algorithm based on the reconstruction of the received signal the modified algorithm; where the data matrix is:

$$
\mathrm{Y}=\mathrm{T} \mathrm{X}^{*}
$$

'*' represents complex conjugate of the received signal, $\mathrm{T}$ is an $\mathrm{N}$ order inverse identity matrix which is called transition matrix. The covariance matrix of the data $\mathrm{Y}$ is :

$$
\mathrm{R}_{\mathrm{Y}}=\mathrm{TR}_{\mathrm{X}}{ }^{*} \mathrm{~T}
$$

We introduce a new array covariance matrix, which is the sum of $R_{Y}$ and $R_{x}$

$$
\begin{aligned}
& \mathrm{R}=\mathrm{R}_{\mathrm{Y}}+\mathrm{R}_{\mathrm{X}} \\
& =\mathrm{AR}_{\mathrm{SA}}+\mathrm{T}\left[\mathrm{AR}_{\mathrm{S}} \mathrm{A}\right]^{*} \mathrm{~T}+2 \sigma^{2} \mathrm{I}
\end{aligned}
$$

By using the matrix theory, if $\mathrm{q}$ is an eigenvector corresponding to a zero eigen value of matrix $A_{S} A$, then $q$ must also be an eigenvector correspond to the zero eigen value of matrix $\mathrm{T}\left[\mathrm{AR}_{\mathrm{S}} \mathrm{A}\right] * \mathrm{~T}$. We observe that matrix $\mathrm{Rx}, \mathrm{RY}$ and $\mathrm{R}$ have the same noise subspace. By performing eigen value decomposition with $\mathrm{R}$, we get its eigen values and its eigen vectors. According to the estimated number of signal sources, the noise subspace among the eigen vectors can be distingue. With the new noise subspace, we can construct MUSIC spatial spectrum:

$$
\mathrm{P}_{\text {MUSIC }}(\theta, \varphi)=\frac{1}{\left(\mathrm{~A}(\theta, \varphi)^{\mathrm{H}} \mathrm{q}_{\mathrm{n}} \mathrm{q}_{\mathrm{n}}{ }^{\mathrm{H}} \mathrm{A}(\theta, \varphi)\right)}
$$

\section{SIMULATION AND COMPARATIVE RESULTS}

In this section, numerical results are presented to analyze the proposed MUSIC algorithm investigated at this research and compared with the experimented one [17-19]. The comparison was made in the same condition: Simulations with 500 independent Monte Carlo methods have been conducted on UCR composed of 9 sensors with half wavelength apart along both $\mathrm{x}$ and $\mathrm{y}$ axis to assess the performance of the proposed estimator. It is assumed that there are $\mathrm{M}=3$ sources at angles $\left(10^{\circ}, 20^{\circ}\right),\left(30^{\circ}, 35^{\circ}\right)$ and $\left(40^{\circ}, 150^{\circ}\right)$ are implying on the array from far-field [17]. A 
uniform circle array (UCA) with five antennas, radius $\mathrm{r}=124$ $\mathrm{mm}$. The carrier frequency is $6 \mathrm{GHz}$. And according to the estimated received signal, the receiving data SNR is above 20 $\mathrm{dB}$ according to [18] and UCA with 8 antenna element, 2 source and noise $=-12 \mathrm{~dB}$, with BPSK modulation, the search step of MUSIC is $0.1^{\circ}$ and the noise intensity is $-12 \mathrm{~d} \mathrm{~B}$ according to [19].

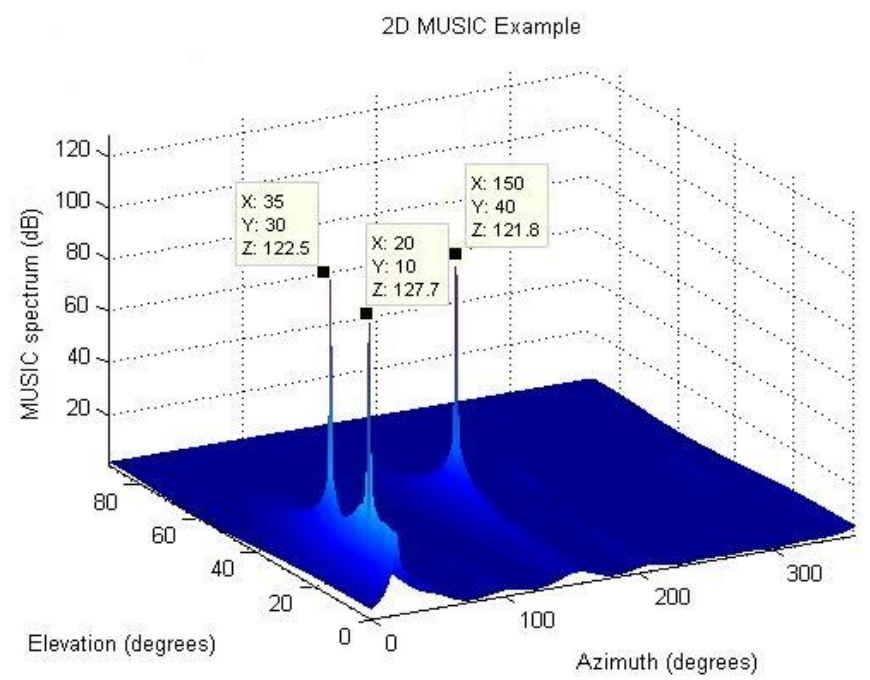

Figure 2: Simulation results for azimuth and elevation $\left(10^{\circ}, 20^{\circ}\right)\left(30^{\circ}, 35^{\circ}\right)$ and $\left(40^{\circ}, 150^{\circ}\right)$

In Figure 2 we plot the spectrums of three functions including 2D-MUSIC, RD-MUSIC [17], and the proposed method. It is seen from this figure that the proposed method estimates correctly the angles: $\theta=\left[10^{\circ} ; 30^{\circ} ; 40^{\circ}\right]$ and $\varphi=\left[20^{\circ} ; 35^{\circ}\right.$; $150^{\circ} \mathrm{]}$. This indicates that we can be estimated the direction of arrival by using the proposed method in 2-D spectral search efficiently, with a lower complexity as compared to 2D-MUSIC indicated in [18].

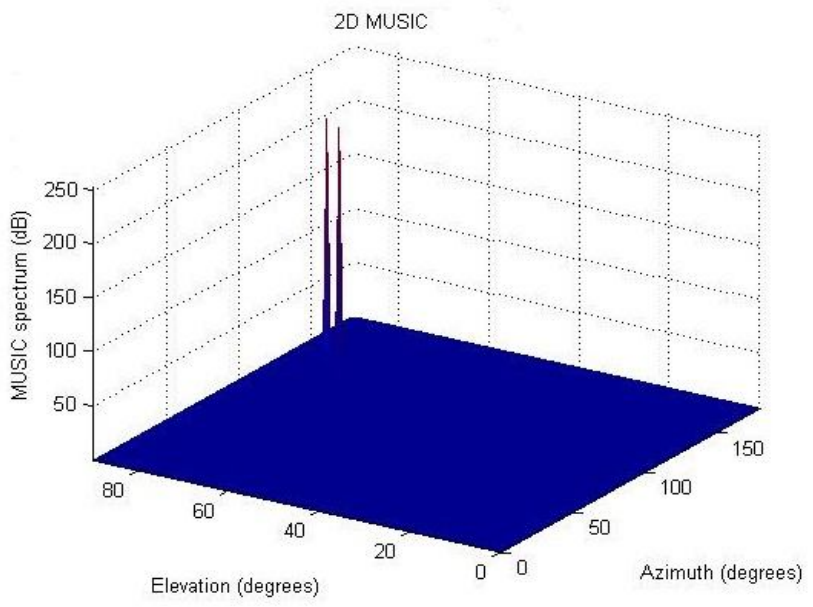

Figure 3: Simulation results for azimuth and elevation $\left(133.6^{\circ}, 137.8^{\circ}\right)$ and $\left(78.6^{\circ}, 82.4^{\circ}\right)$
In Figure 3, we note that the proposed method can resolve clearly the azimuth elevation $\left(133.6^{\circ}, 137.8^{\circ}\right)$ and $\left(78.6^{\circ}, 82.49\right.$ respectively and the peaks are sharp, while the Music only fond one peak around there.

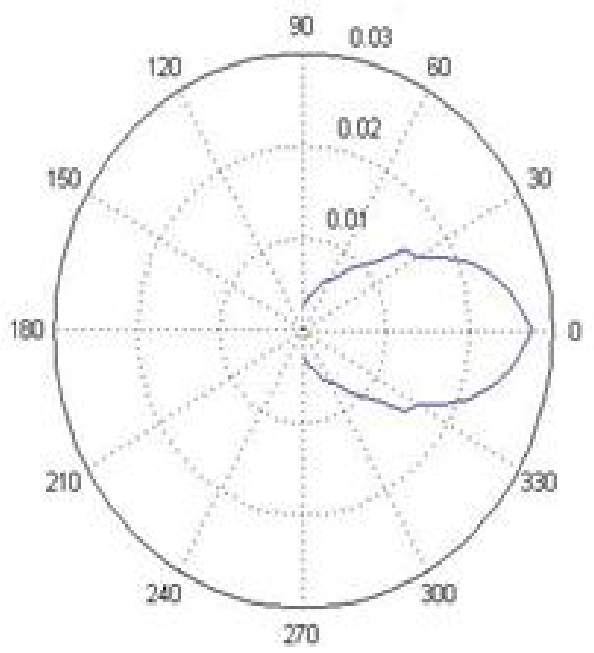

Figure 4:Radiation patterns for the UCA geometry for low SNR

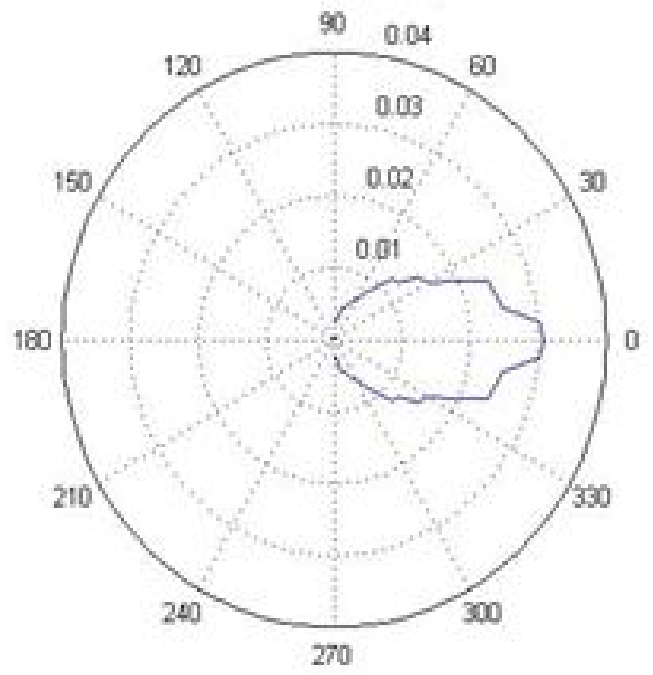

Figure 5.Radiation patterns for the UCA geometry for high SNR

From Figures 4 and 5, we varied the value of the SNR from $-12 \mathrm{~dB}$ to $12 \mathrm{~dB}$ in order to display the influence of this parameter, in the directivity of the Circular Network. According to the figures 4 and 5, we note that the main lobe attenuated but the directivity of the network remains the same, and then our circular network algorithm is able to deal with the case where the signal was very noisy. 
Table 1: Angles of arrival results for different signals

\begin{tabular}{|c|c|c|c|c|c|c|c|c|c|}
\hline Radius & \multicolumn{3}{|c|}{ Proposed Method } & \multicolumn{2}{|c|}{$\begin{array}{c}\text { Proposed Method } \\
\text { Error }\left({ }^{\circ}\right)\end{array}$} & \multicolumn{2}{|c|}{ [19] } & \multicolumn{2}{|c|}{$\begin{array}{c}\text { Error }\left({ }^{\circ}\right) \\
{[19]}\end{array}$} \\
\hline $0.1 \lambda$ & -38.000000 & & 57.000000 & 0 & +0.00000 & -38.000000 & 57.100002 & 0 & +0.10002 \\
\hline $0.5 \lambda$ & -37.899998 & & 57.000002 & $-0,100002$ & +0.00002 & -37.899998 & 57.100004 & $-0,100002$ & +1.00004 \\
\hline $1.0 \lambda$ & -38.000000 & & 57.000000 & 0 & 0 & -38.000000 & 57.000004 & 0 & +0.00004 \\
\hline
\end{tabular}

Table 1 shows the comparison results between the proposed method and the proposed one indicated in [19] for the angles $\theta=-38^{\circ}$ and $\varphi=57^{\circ}$, we resolve that even if the values of number radius change, the proposed method give a higher precision and value of peaks, contrary to experimented one, it cannot detect all angles even if number of signals increases. Results indicate that proposed method MUSIC based on UCA does not have a problem detection for the DOAs with a minimum error which equals $0 \%$ and a maximum equals to $0.2 \%$ for the angle $\theta=-38^{\circ}$ and $0 \%$ to $0.2 \%$ for angle $\varphi=57^{\circ}$ compared to the results published in [19].

\section{CONCLUSION}

This study has introduced the 2-D DOA estimation algorithm for Uniform circular array. The MUSIC uses the eigen values and eigen vectors of the signal and noises to estimate the direction of arrival of the incoming signals. It becomes easier to separate the signals from noise as the eigen vectors for signal and noise subspace are orthogonal to one another. It works efficiently when the signals that are being incident on the array of sensors are non-coherent. Efficiency of this estimation algorithm can be improved by increasing the inter element spacing, increasing the number of antenna sensors, number of snapshots and improving the incidence angle difference between the incoming signals. Moreover we have put forward a modified MUSIC algorithm using the method of reconstructing the data covariance matrix to reduce the algorithm takes full advantage of geometrical norm of UCA, the simulation results and the correlations among the incident signals the proposed comparison have testified the effectiveness of this algorithm. This method provides a new access to estimate DOA with high precision.

\section{REFERENCES}

1. H. Krim and M. Viberg, "Two decades of array signal processing research: the parametric approach," IEEE Sign. Proc. Magn, vol. 13, pp. 67-94, 1996. https://doi.org/10.1109/79.526899

2. C. Thiripurasundari, V. Sumathy, C. Thiruvengadam. "An FPGA implementation of novel smart antenna algorithm in tracking systems for smart cities". vol.2, pp.1-8, 2017.

3. E.Trinidad, L.Materum. "Juxtaposition of Extant TV White Space Technologies for Long-Range Opportunistic Wireless Communications,'International .Jour. Emer. Tren. Engi. Rese (IJETER), Vol. 7,No.8, pp. 210-215 , 2019. https://doi.org/10.30534/ijeter/2019/17782019

4. J.M.Ladrido et all. "Comparative Survey of Signal Processing and Artificial Intelligence Based Channel Equalization Techniques and Technologies," Inte. Jour. Emer. Tren. Engi. Rese (IJETER), Vol. 7,No.9, pp. 311-322, 2019. https://doi.org/10.30534/ijeter/2019/14792019

5. A.Bendali , S. Bri , M. Habibi , M.N. Srifi, A. El fellahi. "Study and Design a Novel Adaptive Array Antenna for a RFIDApplications,'Inte.Jour.Emer.Tren.Engi.Rese (IJETER), Vol.7,No12,pp. 805-811, 2019.

6. B.Liao and S.C. Chan. "DOA Estimation of Coherent Signals for Uniform Linear Arrays with Mutual Coupling," IEEE Proc. Inter. Symp. Circ. Syst. Rio de Janeiro, Brazil, pp. 377-380, 2011. https://doi.org/10.1109/ISCAS.2011.5937581

7. J.G.Nam, S.H.Lee and K.K.Lee. "2-D Nominal Angle Estimation of Multiple Coherently Distributed Sources in a Uniform Circular Array," IEEE ante.wire.prop.lette, vol. 13, pp. 415-418.2014. https://doi.org/10.1109/LAWP.2014.2308191

8. S. S. Balabadrapatruni, "Performance Evaluation of Direction of Arrival Estimation Using Matlab, " Signal \& Image Processing. Int. J; vol 3, pp.57-72. 2012. https://doi.org/10.5121/sipij.2012.3505

9. Z. Wang and S. A. Zekavat, "Manet localization via multi-node TOA-DOA optimal fusion," in Proc. Milcom, 2006, pp. 542-548. 
Adiba EL Fadl et al., International Journal of Emerging Trends in Engineering Research, 8(4), April 2020, 1051 - 1055

10. O. Salychev, "Inertial systems in navigation and geophysics," Bauman Moscow State Technical University Press, Tech. Rep., 1998.

11. M.A. Ihedrane, S.Bri and El.F.Adiba. "High Resolution Method Using Patch Circular Array," International Journal of Electrical and Computer Engineering (IJECE).Vol.7.no4.2017. https://doi.org/10.11591/ijece.v7i4.pp2116-2124

12. M. A. Ihedrane and S.Bri. "Direction of arrival estimation using MUSIC, ESPRIT and maximum-likelihood algorithms for antenna arrays," Walailak J. Sci. Tech., vol. 13, no. 6, pp. 491-502, 2015.

13. S. Yun, K. Kim and S. Nam, "Outer-wall loop antenna for ultra-wide band capsule endoscope system," IEEE Antennas Wireless Propag. Lett., vol. 9, pp. 1135-1138, 2010.

14. M. A. Ihedrane and S.Bri. "2-D MUSIC Algorithm Based on Uniform Triangular Array" 5th International Congress on Information Science and Technology (CiSt), pp. 531-536, 2018.
15. K. Kim, S. Yun, S. Lee, S. Nam, Y. J. Yoon and C. Cheon, “A design of a high-speed and high-efficiency capsule endoscopy system," IEEE Trans. Biomed. Eng., vol. 59, no. 4, pp. 1005-1011, 2012.

https://doi.org/10.1109/TBME.2011.2182050

16. K. Arshak and F. Adepoju, "Adaptive linearized methods for tracking a moving telemetry capsule," in Proc. ISIE, 2007, pp. 2073-2708.

https://doi.org/10.1109/ISIE.2007.4375035

17. W. J. SI, X.Y. LAN and Y. ZOU. "Novel high-resolution DOA estimation using subspace projection method" J. Chi. Uni. Pos. Tel, Vol.19, pp.110-16, 2012.

18. T. Varum, J. N. Matos and P. Pinho.'Direction of Arrival Estimation Analysis Using a 2D Antenna Array," Proce. techn, Vol 17, pp.667-624,2013.

https://doi.org/10.1016/j.protcy.2014.10.267

19. B.Sun. "MUSIC Based on Uniform Circular Array and Its Direction Finding Efficiency,"Inte. J. Sign. Proce. Syst, Vol 1, pp.273-277, 2013.

https://doi.org/10.12720/ijsps.1.2.273-277 